\title{
Adaptive optics, near-infrared observations of magnetars
}

\author{
V. Testa ${ }^{1}$, N. Rea ${ }^{2,3}$, R. P. Mignani ${ }^{4}$, G. L. Israel ${ }^{1}$, R. Perna ${ }^{5}$, S. Chaty ${ }^{6}$, L. Stella ${ }^{1}$, S. Covino ${ }^{7}$, R. Turolla ${ }^{8,4}$, \\ S. Zane ${ }^{4}$, G. Lo Curto ${ }^{9}$, S. Campana ${ }^{7}$, G. Marconi ${ }^{9}$, and S. Mereghetti ${ }^{10}$ \\ 1 INAF - Osservatorio Astronomico di Roma, via Frascati 33, 00040 Monte Porzio Catone, Italy \\ e-mail: testa@mporzio.astro.it \\ 2 University of Amsterdam, Astronomical Institute "Anton Pannekoek", Kruislaan 403, 1098 SJ, The Netherlands \\ 3 SRON Netherlands Institute for Space Research, Sorbonnelaan, 2, 3584 CA, Utrecht, The Netherlands \\ ${ }^{4}$ MSSL, University College London, Holmbury St. Mary, Dorking Surrey RH5 6NT, UK \\ 5 JILA and Department of Astrophysical and Planetary Sciences, University of Colorado, 440 UCB, Boulder, CO, 80309, USA \\ ${ }^{6}$ Laboratoire AIM, CEA/DSM - CNRS - Université Paris Diderot, DAPNIA/Service d'Astrophysique, Bât.709, CEA-Saclay, \\ 91191 Gif-sur-Yvette, France \\ 7 INAF - Osservatorio Astronomico di Brera, via Bianchi 46, 23807 Merate (Lc), Italy \\ 8 University of Padua, Physics Department, via Marzolo 8, 35131, Padova, Italy \\ 9 ESO - European Southern Observatory, Alonso de Cordova 3107, Vitacura, Santiago, Chile \\ 10 INAF - Istituto di Astrofisica Spaziale e Fisica Cosmica "G.Occhialini", via Bassini 15, 20133, Milano, Italy
}

Received 17 September 2007 / Accepted 17 January 2008

\section{ABSTRACT}

\begin{abstract}
Context. We report on near-infrared (IR) observations of the three anomalous X-ray pulsars XTE J1810-197, 1RXS J1708-4009, and 1E 1841-045, and the soft gamma-ray repeater SGR 1900+14, taken with the ESO-VLT, the Gemini, and the CFHT telescopes.

Aims. This work aims at identifying and/or confirming the IR counterparts of these magnetars, as well as at measuring their possible IR variability.

Methods. To perform photometry of objects as faint as $K_{\mathrm{s}} \sim 20$, we used data taken with the largest telescopes, equipped with the most advanced IR detectors and in most of the cases with adaptive optics devices. The latter are critical for achieving the sharp spatial accuracy required to pinpoint faint objects in crowded fields.

Results. We confirm with high confidence identification of the IR counterpart to XTE J1810-197 and its IR variability. For 1E 1841045 and SGR 1900+14, we propose two candidate IR counterparts based on the detection of IR variability. For 1RXS J1708-4009, we show that none of the potential counterparts within the source X-ray error circle can as yet be convincingly associated with this AXP. Conclusions. The IR variability of the AXP XTE J1810-197 does not follow the same monotonic decrease in its post-outburst X-ray emission. Instead, the IR variability appears more like the one observed in radio band, although simultaneous IR and radio observations are crucial for any conclusion in this respect. For 1E 1841-045 and SGR 1900+14, follow-up observations are needed to confirm our proposed candidates with higher confidence.
\end{abstract}

Key words. stars: pulsars: individual: XTE J1810-197 - stars: pulsars: individual: 1RXS J1708-4009 -

stars: pulsars: individual: 1E 1841-045 - stars: pulsars: individual: SGR 1900+14 - stars: pulsars: general - stars: neutron

\section{Introduction}

In recent years, the study of isolated neutron stars has become one of the most challenging research areas in high-energy astrophysics, largely as a result of the discovery of several new classes of sources besides the classical, well-studied radio pulsars. The most extreme objects are the so-called "magnetars". This class comprises the anomalous X-ray pulsars (AXPs) and the soft gamma-ray repeaters (SGRs), observationally very similar in many respects. They all are slow X-ray pulsars with periods in a narrow range $(P=2-12 \mathrm{~s})$, relatively largeperiod derivatives $\left(\dot{P}=10^{-13}-10^{-10} \mathrm{~s} \mathrm{~s}^{-1}\right)$, spin-down ages of $10^{3}-10^{4} \mathrm{yr}$, and magnetic fields, as inferred from the classical magnetic dipole spin-down formula ${ }^{1}$, of $10^{14}-10^{15} \mathrm{G}$, hence larger than the electron quantum critical field $\left(B_{\mathrm{cr}} \simeq 4.4 \times\right.$ $\left.10^{13} \mathrm{G}\right)$ above which quantum effects become crucial. Both AXPs and SGRs are strong X-ray emitters, with X-ray luminosities of about $10^{34}-10^{36} \mathrm{erg} \mathrm{s}^{-1}$. Their $0.1-10 \mathrm{keV}$ persistent

${ }_{1} B \sim 3 \times 10^{19} \sqrt{P \dot{P}} \mathrm{G}$. emission has relatively soft spectra usually modelled by an absorbed blackbody $(k T \sim 0.2-0.6)$ plus a power law $(\Gamma \sim 2-4$; for a review see, e.g., Woods \& Thompson 2006). Their X-ray energy output is much greater than the rotational energy loss and implies that these sources are not rotationally powered, at variance with most young isolated neutron stars. Rather, AXPs and SGRs are believed to be powered by the neutron star ultrastrong magnetic field (Duncan \& Thompson 1992; Thompson \& Duncan 1995). In the "magnetar" model, crustal deformations, driven by magnetic stresses imparted to the crust by the strong internal toroidal magnetic field, are responsible for the observed activity, $\mathrm{X} / \gamma$-ray bursts, and giant flares.

Until not long ago, the persistent emission of AXPs and SGRs was only detected in the soft X-ray range. However, in the past few years the availability of more sensitive optical and IR telescopes, as well as of $\gamma$-ray satellites, opened new windows on the study of AXPs and SGRs, unveiling their multiband properties. In particular, observations from ESO, Gemini, CFHT, and Keck (see Israel et al. 2004a, for a review) led to the discovery of faint $\left(K_{\mathrm{S}} \sim 20\right)$ and, in some cases variable, 
Table 1. Observations summary.

\begin{tabular}{|c|c|c|c|c|c|c|c|}
\hline Telescope/Instrument & Target & Date (YY.MM.DD) & MJD & Filter & Exposure time (s) & $F W H M\left({ }^{\prime \prime}\right)$ & airmass \\
\hline \multirow[t]{14}{*}{ VLT/NACO } & XTEJ1810-197 & 2003.10 .08 & 52920 & $H$ & 2520 & 0.189 & 1.17 \\
\hline & & 2003.10.08 & 52920 & $K_{\mathrm{s}}$ & 2520 & 0.243 & 1.49 \\
\hline & & 2004.03 .14 & 53078 & $\dot{H}$ & 1080 & 0.108 & 1.47 \\
\hline & & 2004.03.14 & 53078 & $K_{\mathrm{s}}$ & 1200 & 0.094 & 1.20 \\
\hline & & 2004.03 .14 & 53078 & $J$ & 1080 & 0.081 & 1.13 \\
\hline & & 2004.09.09 & 53257 & $J$ & 1800 & 0.170 & 1.01 \\
\hline & & 2004.09.09 & 53257 & $H$ & 3600 & 0.140 & 1.08 \\
\hline & & 2004.09.13 & 53261 & $K_{\mathrm{s}}$ & 3600 & 0.099 & 1.01 \\
\hline & 1RXS J1708-4009 & 2003.05 .20 & 52779 & $K_{\mathrm{s}}$ & 2400 & 0.083 & 1.04 \\
\hline & & 2003.06 .19 & 52809 & $H$ & 2400 & 0.122 & 1.40 \\
\hline & & 2003.06 .20 & 52810 & $J$ & 2400 & 0.122 & 1.04 \\
\hline & & 2004.03 .25 & 53089 & $L^{\prime}$ & 2100 & 0.119 & 1.04 \\
\hline & SGR 1900+14 & 2006.04 .01 & 53826 & $K_{\mathrm{s}}$ & 1560 & 0.125 & 1.43 \\
\hline & & 2006.07.21 & 53937 & $K_{\mathrm{s}}$ & 2040 & 0.111 & 1.21 \\
\hline \multirow[t]{8}{*}{ Gemini/NIRI } & XTE J1810-197 & 2003.09 .18 & 52900 & $K_{\mathrm{s}}$ & 285 & 0.397 & 1.62 \\
\hline & & 2003.09 .18 & 52900 & $H$ & 285 & 0.397 & 1.99 \\
\hline & & 2003.09 .18 & 52900 & $J$ & 285 & 0.397 & 1.78 \\
\hline & & 2004.06.10 & 53166 & $K_{\mathrm{s}}$ & 510 & 0.631 & 1.32 \\
\hline & & 2004.06.10 & 53166 & $H$ & 510 & 0.526 & 1.30 \\
\hline & & 2004.06.10 & 53166 & $J$ & 510 & 0.655 & 1.32 \\
\hline & & 2004.07.28 & 53214 & $K_{\mathrm{s}}$ & 510 & 0.468 & 1.39 \\
\hline & & 2004.07.28 & 53214 & $H$ & 510 & 0.526 & 1.53 \\
\hline \multirow[t]{2}{*}{ CFHT/AOBIR } & 1E 1841-045 & 2002.08 .17 & 52503 & $K^{\prime}$ & 1260 & 0.137 & 1.11 \\
\hline & & 2002.08 .17 & 52503 & $H$ & 1260 & 0.161 & 1.31 \\
\hline
\end{tabular}

IR counterparts to five out of seven confirmed AXPs (one of which was also detected in the optical band by Hulleman et al. (2000), and to one (out of four) confirmed SGR (Israel et al. 2005; Kosugi et al. 2005). Furthermore, deep INTEGRAL observations in the $20-200 \mathrm{keV}$ band revealed that most of these highly magnetized sources are also hard X-ray emitters (Kuiper et al. 2004; Mereghetti et al. 2005; Kuiper et al. 2006; Götz et al. 2006). The recent discovery of pulsed radio emission from the transient magnetars XTEJ1810-197 (Halpern et al. 2005; Camilo et al. 2006) and 1E 1547.0-5408 (Camilo et al. 2007c), the only cases so far (Burgay et al. 2006, 2007), is intriguing since radio emission was believed to be quenched in magnetic fields above the quantum critical limit (Baring \& Harding 1998).

Despite the more complete observational picture we now have for AXPs and SGRs, the physical processes at the basis of their emission in the different bands are not fully understood as yet. This is particularly true as far as the optical and IR emission of these sources is concerned. The extrapolation of the canonical blackbody plus power law, used to model the soft $\mathrm{X}$-ray emission of these sources, largely overpredicts their optical and IR emission. Recently, new attempts have been made at modelling the multi-band spectra of magnetars to overcome this problem. In particular, Rea et al. (2007a) showed that AXP spectra can be fitted by a resonant cyclotron scattering model or two log-parabolic functions, which do not overpredict their dim optical and IR magnitudes. However, despite solving this issue, these new spectral models are still far from giving any physical interpretation for the optical and IR emission.

Some possibilities are that either the magnetars optical/IR emission arise from the star surface/magnetosphere (Beloborodov \& Thompson 2007) or from the reprocessing of the X-ray emission via a fossil disk around the neutron star (Chatterjee et al. 2000; Perna et al. 2000a). Indeed, the Spitzer mid-IR detection of the AXP 4U 0142+614(Wang et al. 2006) has been interpreted as first proof of a passive fossil disk around an AXP, believed to emit through reprocessing of the X-ray radiation from the magnetar.
In this paper we report on Gemini and ESO-VLT observations of the transient AXP XTE J1810-197, on ESO-VLT observations of the AXP 1RXS J1708-4009 and of SGR 1900+14, and CFHT observations of the AXP 1E 1841-045 (Sect. 2). The results are presented and discussed in the context of different emission scenarios and compared with the optical and IR properties of other magnetars (see Sect. 4).

\section{Observations and data reduction}

\subsection{Observations}

Observations were performed with the $8 \mathrm{~m}$ Very Large Telescope (VLT) and the $3.5 \mathrm{~m}$ Canada-France-Hawaii Telescope (CFHT), both equipped with adaptive optics (AO) and near-IR cameras. In addition, we used archival observations performed with the $8 \mathrm{~m}$ Gemini telescope, also equipped with an IR camera but without AO, which were offered only starting from 2004. A summary of the telescopes and instruments used, as well as the observations $\operatorname{logs}$, is reported in Table 1 . In all cases, images were obtained through the standard observing technique commonly applied to IR arrays, i.e. by stacking repeated exposures along each node of a pre-defined dithering pattern, with each node jittered by a few arcsec with respect to the previous one. This allowed us to obtain a set of dithered exposures that are used to produce sky-background images.

VLT/Yepun observations of XTE J1810-197, 1RXS J17084009, and SGR 1900+14 were carried out in different observing runs (see Table 1) using NAos COnica (NACO) ${ }^{2}$ with the S27 camera (0.'027 pixel size, $28^{\prime \prime} \times 28^{\prime \prime}$ field of view). Gemini-North observations of XTE J1810-197 were carried out using the Near-InfraRed Imager (NIRI) ${ }^{3}$ with the $\mathrm{f} / 6$ camera $\left(0{ }^{\prime} 117\right.$ pixel scale, $120^{\prime \prime} \times 120^{\prime \prime}$ field of view). $1 \mathrm{E} 1841-045$ was

\footnotetext{
2 wWw.eso.org/instruments/naco

3 wwW.gemini.edu/sciops/instruments/niri
} 
observed at the CFHT using the Adaptive Optics Bonnet (AOB $)^{4}$ InfraRed camera (0.'035 pixel size, 36" $\times 36^{\prime \prime}$ field of view).

\subsection{Data reduction and calibration}

The VLT data were pre-reduced with the ESO NACO pipeline ${ }^{5}$, which is based on the package eclipse, while the native version of the eclipse $e^{6}$ package was used for the Gemini and CFHT data. In all cases, the image pre-reduction produced a final co-added, sky-subtracted and flat-fielded image for each band.

Depending on the case, fluxes were computed through either PSF or aperture photometry using the $\mathrm{IRAF}^{7}$ version of the daophot package (Stetson 1992). For AO observations we found some instrument-dependent effects that hampered the PSF fitting and discouraged the use of PSF photometry. In particular, the PSF was found to be variable across the field of view in the VLTNACO images, while in the CFHT-AOB images it was found to feature an unusual profile with a clearly visible secondary diffraction ring. Both effects are difficult to account for, even by using more sophisticated and position-dependent PSF models. On the other hand, the PSF was found to be more stable in the Gemini-NIRI images, although worse sampled due to the larger pixel size. For these reasons, we decided to use aperture photometry as a general strategy for our AO images, i.e. the NACO and AOB ones, and to use PSF photometry for the NIRI images. For the former, aperture photometry was performed using an aperture or diameter twice the measured PSF. Aperture and PSF photometries were compared by applying aperture corrections using the IRAF daogrow algorithm, which calculates the aperture correction extrapolating to an ideally infinite aperture.

For the VLT images, the photometric calibration was performed by observing standard stars from the Persson et al. (1998) catalogue, usually observed under similar conditions (and at about the same epoch) as our targets. For all the NACO runs, the zeropoint uncertainty was found to be very small, i.e. in the 0.02-0.05 mag range. The residual uncertainty introduced by the extinction term is almost wiped out by the closeness in time and airmass of target fields and standards. Since it turns out to be $\$ 0.01 \mathrm{mag}$, it has been neglected.

The Gemini images of XTE J1810-197 were at first instance calibrated with the available standard stars, but the uncertainty in the zero points and the zero point differences usually found between standards taken on the same nights suggested discarding the primary standard calibration. A photometric calibration was also tried using stars from the 2MASS (Skrutskie et al. 2006) catalogue identified in the target fields. However, such an on-theframe calibration suffered from two main problems. First, the number of suitable 2MASS stars was often quite small and the brighter ones were found to be generally saturated in our images. Furthermore, the 2MASS catalogue was built from images with a $2^{\prime \prime}$ pixel size so that, in many cases, the measured magnitudes corresponded to blended objects that are, instead, resolved in our higher resolution images. Thus, given the accuracy of the NACO zeropoints and the very low rms of the photometric solution, we chose to re-calibrate the Gemini photometry of the XTEJ1810197 field on the NACO one by using, as secondary photometric

\footnotetext{
4 wWw.cfht.hawaii.edu/Instruments

5 www.eso.org/instruments/naco/

6 www.eso.org/eclipse

7 IRAF is distributed by the National Optical Astronomy Observatories, which are operated by the Association of Universities for Research in Astronomy, Inc., under cooperative agreement with the National Science Foundation.
}

calibrators, a set of well-suited field stars in common between the NACO and the NIRI images. As a reference, we chose the 2004 September NACO observations because of their overall better quality. To ensure full consistency among observations performed at different epochs (Table 1), also the NACO 2003 October and 2004 March photometry of the XTE J1810197 field was re-calibrated to the 2004 September one. The relative photometric uncertainty, based on about 100 stars in common among the various images is of the order of $\approx 0.05$ mag for the NACO-to-NACO registration, and slightly worse for the Gemini-to-NACO one ( $\approx 0.08 \mathrm{mag})$.

For the CFHT photometry, for which no NACO images are available, the only option was to use the 2MASS catalogue, after carefully accounting for all the caveats mentioned above. For the $L^{\prime}$ observation of 1RXS J1708-4009, we used standard stars from the UKIRT IR standard catalogue (Leggett et al. 2003), yielding a photometric accuracy of $\sim 0.1 \mathrm{mag}$.

In all cases, astrometric calibration was performed using 2MASS stars as the reference, yielding an average uncertainty of $\sim 0$ ' 2 , after accounting for the intrinsic astrometric accuracy of 2MASS ( 200 mas) and the rms of the astrometric fits. However, due to the small instrument pixel scales, the latter was found to be much smaller than the 2MASS astrometric accuracy, so it has been neglected.

\section{Data analysis and results}

\subsection{XTE J1810-197}

Figure 1 (left) shows the XTEJ1810-197 field ( $K_{\mathrm{S}}$ band) observed with NACO on October 2003 (see also Israel et al. 2004b). The XTE J1810-197 candidate IR counterpart, labelled A, is coincident with the radio position (RA $18^{\mathrm{h}} 09^{\mathrm{m}} 51^{\mathrm{s}} .087$, Dec $-19^{\circ} 43^{\prime} 51^{\prime \prime}$ '93; error $=0$ O' $^{\prime} 01$; Camilo et al. 2006). For both the NACO and NIRI observations, photometry was computed and calibrated as described in the previous section. For each instrument, the resulting $J, H$, and $K_{\mathrm{s}}$ band catalogues were then matched and compared with the multi-band ones. The multiband magnitudes of the XTE J1810-197 candidate counterpart, all calibrated to the NACO system, are reported in Table 2, together with those of a number of field objects, chosen among those used as a reference for the photometry cross-calibrations (see previous section). We note that the candidate counterpart is not detected in the $J$ and $K_{\mathrm{s}}$-band Gemini observations of the 2004 June run, which were performed in non-photometric conditions. Given the short integration time, the overall poor data quality, and the lack of standard observations, we could only derive tentative estimates on the limiting magnitudes ( $3 \sigma$ upper limits of $J>21.9$ and $K_{\mathrm{s}}>20$ ), which are of no use in constraining the flux evolution of our source.

The time-averaged $\left(K_{\mathrm{s}}, H-K_{\mathrm{s}}\right)$ colour-magnitude diagram (CMD) of the field is shown in Fig. 1 (right). A well-defined main sequence group of stars, probably tracking a young star cluster, is recognisable in the left part of the diagram, while a second red clump of stars, most likely red giants or highly absorbed main sequence stars, is present on the right. The XTE J1810-197 counterpart (green star) appears much fainter than the nearby comparison stars (red dots), and its colour indeed suggests that it might be a peculiar object.

The variability of the XTEJ1810-197 IR counterpart is shown in Fig. 2, together with the X-ray variability of the X-ray source (see Sect. 4 for details). While the source features a clear, almost monotonic flux decay in the X-rays, in the IR there is no clear trend in the flux evolution, hence no correlation with the 

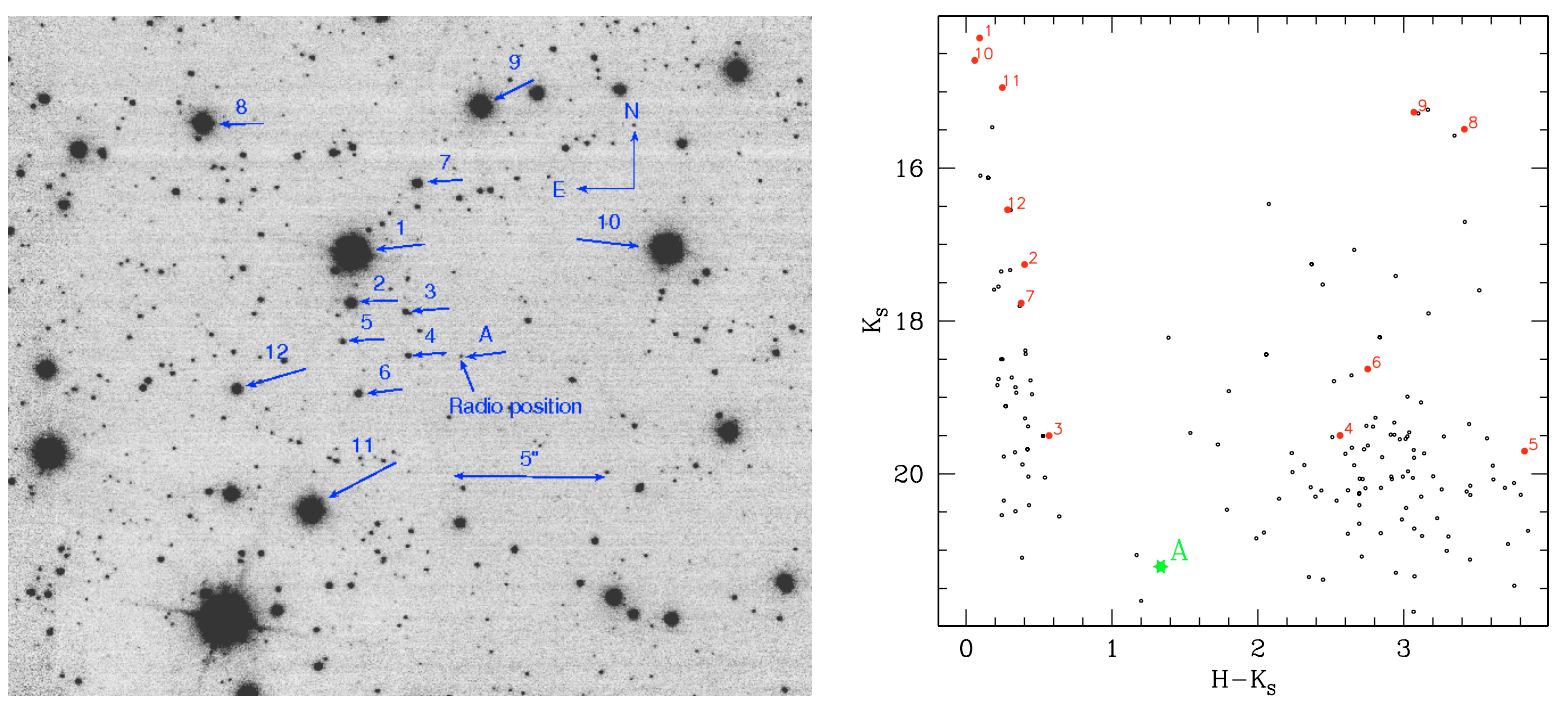

Fig. 1. XTE J1810-197: Left panel: VLT/NACO K-band image (March 2004). The radio position (Camilo et al. 2007c) is marked with its candidate counterpart (object A). Twelve nearby objects, some of them used as a reference for relative photometry, are also marked in the figure. Right panel: $K_{\mathrm{S}}$ vs. $H-K_{\mathrm{S}}$ colour-magnitude diagram of the sources detected in the NACO field of view (filled circles). Object A is marked with a rectangle indicating the range in colour and magnitude covered throughout the epochs, while some of the nearby objects marked in the map and listed in Table 2 are plotted in red and labeled. Magnitudes and colours for all the sources but object "A" have been averaged over all the observations.

X-rays. Indeed, the flux seems to vary in an erratic way in all three IR bands. In particular, we find that the overall variability is apparently greater in the $H$ and $J$ bands $(1.07 \pm 0.24$ and $0.97 \pm$ $0.32 \mathrm{mag}$, respectively) than in the $K_{\mathrm{s}}$ one $(0.40 \pm 0.13 \mathrm{mag})$. We note that our detailed re-analysis of the 2003 October and 2004 March observations of XTE J1810-197 yields a $K_{\mathrm{s}}$ band variability slightly smaller $(0.36 \pm 0.10)$ than originally reported by Rea et al. (2004) using the same observations $(0.50 \pm 0.10)$, although it is still consistent within the errors. We attribute this (non significant) difference to the more accurate relative photometry between the two epochs, performed using a larger set of secondary photometric calibrators than the one used in Rea et al. (2004). The last panel shows the $\left(H-K_{\mathrm{s}}\right)$ colour variability of the source. In general, we note that the source seems to become redder when its flux decreases, although no clear trend can be recognised in our sparse photometry. We need to warn, however, that any conclusion on the source flux and colour variability must be taken with due care. First of all, the apparent lack of correlation between the variability in the $H$ and $K_{\mathrm{s}}$ bands is only suggested by the 2004 July Gemini $K_{\mathrm{s}}$ band observation. If we take this observation out and consider only the co-eval flux measurements, the $K_{\mathrm{S}}$ band lightcurve becomes more consistent with the $H$ (as well as with the $J$ ) band one. More generally, although special care was taken to check the cross-instrument calibration (see Sect. 2.2), it is possible that our relative photometry is affected by random errors. These may be induced, e.g. by fluctuations in the atmospheric conditions, sky background, seeing, quality of the primary calibration frames (dark and flatfields), and glitches in the detector performance. Of course, while these effects are marginal for the relatively bright stars that we have used as a reference to compute our cross-instrument photometric calibration, they are indeed significant for much fainter stars, such as the XTEJ1810-197 candidate counterpart $\left(K_{\mathrm{S}} \sim 21\right)$, and can increase the overall uncertainties on the measured magnitudes. To quantify these random errors we have cross-checked the photometry of a number of test stars with brightness comparable to the one of our target. We found that all test stars show a scatter of $\sim 0.30 \mathrm{mag}$ in $J$ and of $\sim 0.10 \mathrm{mag}$ in the $H$ and $K_{\mathrm{s}}$ bands for the NACO-to-NACO registration, while the scatter increases to $\sim 0.20 \mathrm{mag}$ for the Gemini-to-NACO registration. Hence, we take these values as representative of the random errors of our relative photometry. By adding them to the formal errors, we find that only the $H$ band variability $(1.07 \pm 0.26)$ can be considered formally significant, and it is consistent with that measured in the $J$ band $(0.97 \pm 0.43)$ and, marginally, also with the $K_{\mathrm{s}}$ band one $(0.40 \pm 0.16)$.

\subsection{RXS J1708-4009}

Figure 3 shows the NACO $K_{\mathrm{s}}$-band image of the field around the Chandra position of 1 RXS J1708-4009(RA $17^{\mathrm{h}} 08^{\mathrm{m}} 46^{\mathrm{s}} .9$, Dec $\left.-40^{\circ} 08^{\prime} 52^{\prime \prime} .4\right)$. The size of the X-ray error circle was estimated considering the uncertainty on the Chandra position, that is 0.7 (Israel et al. 2003), combined with the overall accuracy of our astrometric calibration (see Sect. 2.2), yielding a final uncertainty of 1 .' 1 at $99 \%$ confidence level. The magnitudes of all the objects detected within the X-ray error circle are reported in Table 3. No other object is detected down to the $3 \sigma$ limiting magnitudes of 22.98, 22.20, 22.26 in the $J, H, K_{\mathrm{s}}$ bands, respectively. In the $L^{\prime}$ band, no object is detected within the X-ray error circle down to 17.8 (the deepest limit ever obtained for an AXPs in this band). Figure 4 shows the $\left(K_{\mathrm{s}}, J-K_{\mathrm{s}}\right)$ and $\left(H, H-K_{\mathrm{s}}\right)$ CMDs of all the objects detected in the field. The two CMDs show a fairly scattered sequence. Despite previous claims (Israel et al. 2003; Safi-Harb \& West 2005; Durant \& van Kerkwijk 2007), none of these objects show significant $(>3 \sigma)$ IR variability to justify a safe identification as the IR counterpart of 1RXS J1708-4009 (see also Sect. 4).

\section{3. $1 E$ 1841-045}

An updated determination of the 1E 1841-045 position (Wachter et al. 2004) was obtained with Chandra and gives RA $18^{\mathrm{h}} 41^{\mathrm{m}} 19.336$, Dec $-04^{\circ} 56^{\prime} 10^{\prime} .83$, with a $3 \sigma$ uncertainty radius of 0.9 . However, this uncertainty is based only on five 2MASS reference stars used for the boresight correction (Wachter et al. 2004). We decided to use a conservative nominal 
Table 2. $K_{\mathrm{s}}, H$, and $J$ magnitudes for the candidate IR counterpart of XTEJ1810-197 and twelve nearby objects, for the 6 different observing runs.

\begin{tabular}{|c|c|c|c|c|c|c|c|c|c|}
\hline Source Id. & RA (2000) & $\operatorname{Dec}(2000)$ & 52900 & 52920 & 53078 & 53166 & 53214 & 53257 & $53261^{a}$ \\
\hline \multicolumn{10}{|c|}{ 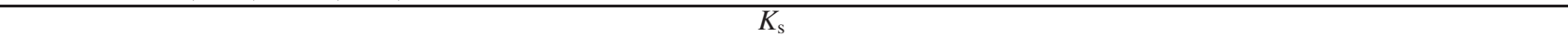 } \\
\hline A & 272.46283 & -19.73110 & $20.81 \pm 0.08$ & $20.82 \pm 0.09$ & $21.18 \pm 0.05$ & \multicolumn{3}{|c|}{$21.21 \pm 0.10$} & $21.04 \pm 0.09$ \\
\hline 1 & 272.46390 & -19.73016 & $13.87 \pm 0.07$ & $13.85 \pm 0.04$ & $14.26 \pm 0.04$ & \multicolumn{3}{|c|}{$14.01 \pm 0.04$} & $14.01 \pm 0.08$ \\
\hline 2 & 272.46391 & -19.73061 & $17.23 \pm 0.07$ & $17.25 \pm 0.04$ & $17.23 \pm 0.04$ & \multicolumn{3}{|c|}{$17.36 \pm 0.04$} & $17.30 \pm 0.08$ \\
\hline 3 & 272.46338 & -19.73070 & $19.09 \pm 0.07$ & $19.21 \pm 0.05$ & $19.47 \pm 0.04$ & \multicolumn{3}{|c|}{$19.15 \pm 0.05$} & $19.41 \pm 0.08$ \\
\hline 4 & 272.46335 & -19.73110 & $19.43 \pm 0.07$ & $19.37 \pm 0.05$ & $19.46 \pm 0.04$ & \multicolumn{3}{|c|}{$19.42 \pm 0.04$} & $19.47 \pm 0.08$ \\
\hline 5 & 272.46399 & -19.73098 & $19.52 \pm 0.07$ & $19.77 \pm 0.06$ & $19.67 \pm 0.04$ & \multicolumn{3}{|c|}{$19.62 \pm 0.04$} & $19.70 \pm 0.08$ \\
\hline 6 & 272.46384 & -19.73145 & $18.61 \pm 0.07$ & $18.61 \pm 0.04$ & $18.60 \pm 0.04$ & \multicolumn{3}{|c|}{$18.66 \pm 0.04$} & $18.67 \pm 0.08$ \\
\hline 7 & 272.46327 & -19.72952 & $17.69 \pm 0.07$ & $17.70 \pm 0.04$ & $17.73 \pm 0.04$ & \multicolumn{3}{|c|}{$17.75 \pm 0.04$} & $17.75 \pm 0.08$ \\
\hline 8 & 272.46535 & -19.72897 & $15.48 \pm 0.07$ & $15.40 \pm 0.04$ & $15.45 \pm 0.04$ & \multicolumn{3}{|c|}{$15.51 \pm 0.04$} & $15.53 \pm 0.08$ \\
\hline 9 & 272.46264 & -19.72882 & $15.25 \pm 0.07$ & $15.15 \pm 0.04$ & $15.23 \pm 0.04$ & \multicolumn{3}{|c|}{$15.27 \pm 0.04$} & $15.23 \pm 0.08$ \\
\hline 10 & 272.46082 & -19.73013 & $14.43 \pm 0.07$ & $14.19 \pm 0.04$ & $14.55 \pm 0.04$ & \multicolumn{3}{|c|}{$14.44 \pm 0.04$} & $14.29 \pm 0.08$ \\
\hline 11 & 272.46430 & -19.73252 & $14.83 \pm 0.07$ & $14.74 \pm 0.04$ & $14.91 \pm 0.04$ & \multicolumn{3}{|c|}{$14.87 \pm 0.04$} & $14.90 \pm 0.08$ \\
\hline 12 & 272.46502 & -19.73141 & $16.59 \pm 0.07$ & $16.50 \pm 0.04$ & $16.51 \pm 0.04$ & \multicolumn{3}{|c|}{$16.63 \pm 0.04$} & $16.54 \pm 0.08$ \\
\hline \multicolumn{10}{|c|}{$H$} \\
\hline A & & & $21.67 \pm 0.12$ & $21.81 \pm 0.16$ & $22.55 \pm 0.07$ & \multicolumn{4}{|c|}{$21.48 \pm 0.12 \quad 21.73 \pm 0.11 \quad 22.29 \pm 0.18$} \\
\hline 1 & & & $14.27 \pm 0.05$ & $14.20 \pm 0.04$ & $14.39 \pm 0.04$ & $14.24 \pm 0.09$ & $14.24 \pm 0.08$ & \multicolumn{2}{|l|}{$14.24 \pm 0.08$} \\
\hline 2 & & & $17.66 \pm 0.05$ & $17.68 \pm 0.04$ & $17.66 \pm 0.04$ & $17.69 \pm 0.09$ & $17.71 \pm 0.08$ & \multicolumn{2}{|l|}{$17.67 \pm 0.08$} \\
\hline 3 & & & $20.00 \pm 0.06$ & $20.11 \pm 0.05$ & $20.07 \pm 0.04$ & $19.90 \pm 0.09$ & $19.78 \pm 0.09$ & \multicolumn{2}{|l|}{$20.07 \pm 0.08$} \\
\hline 4 & & & $22.24 \pm 0.14$ & $22.05 \pm 0.11$ & $22.06 \pm 0.09$ & $21.66 \pm 0.23$ & $21.87 \pm 0.10$ & \multicolumn{2}{|l|}{$22.64 \pm 0.17$} \\
\hline 5 & & & $22.28 \pm 0.28$ & $22.74 \pm 0.42$ & - & $21.79 \pm 0.21$ & $21.68 \pm 0.10$ & \multicolumn{2}{|l|}{$22.80 \pm 0.11$} \\
\hline 6 & & & $21.17 \pm 0.09$ & $21.38 \pm 0.07$ & $21.38 \pm 0.05$ & $21.11 \pm 0.10$ & $21.15 \pm 0.09$ & \multicolumn{2}{|l|}{$21.34 \pm 0.09$} \\
\hline 7 & & & $18.16 \pm 0.06$ & $18.19 \pm 0.04$ & $18.14 \pm 0.04$ & $18.14 \pm 0.09$ & $18.15 \pm 0.08$ & \multicolumn{2}{|l|}{$18.20 \pm 0.08$} \\
\hline 8 & & & $18.95 \pm 0.06$ & $19.00 \pm 0.05$ & $18.90 \pm 0.04$ & $18.90 \pm 0.09$ & $18.93 \pm 0.08$ & \multicolumn{2}{|l|}{$18.95 \pm 0.08$} \\
\hline 9 & & & $18.42 \pm 0.06$ & $18.42 \pm 0.04$ & $18.34 \pm 0.04$ & $18.44 \pm 0.09$ & $18.41 \pm 0.08$ & \multicolumn{2}{|l|}{$18.44 \pm 0.08$} \\
\hline 10 & & & $14.62 \pm 0.05$ & $14.51 \pm 0.04$ & $14.65 \pm 0.05$ & $14.61 \pm 0.09$ & $14.61 \pm 0.08$ & - & \\
\hline 11 & & & $15.15 \pm 0.05$ & $15.12 \pm 0.04$ & $15.19 \pm 0.04$ & $15.13 \pm 0.09$ & $15.14 \pm 0.08$ & $15.12 \pm 0.08$ & \\
\hline 12 & & & $16.91 \pm 0.05$ & $16.89 \pm 0.04$ & $16.83 \pm 0.04$ & $16.93 \pm 0.09$ & $16.91 \pm 0.08$ & $16.90 \pm 0.08$ & \\
\hline & & & & & $J$ & & & & \\
\hline A & & & $22.92 \pm 0.22$ & & $23.89 \pm 0.23$ & & & $23.45 \pm 0.12$ & \\
\hline 1 & & & - & & $15.37 \pm 0.06$ & & & $15.04 \pm 0.04$ & \\
\hline 2 & & & $18.85 \pm 0.05$ & & $18.83 \pm 0.06$ & & & $18.88 \pm 0.04$ & \\
\hline 3 & & & $21.06 \pm 0.06$ & & $21.35 \pm 0.07$ & & & $21.35 \pm 0.04$ & \\
\hline 7 & & & $19.34 \pm 0.05$ & & $19.39 \pm 0.06$ & & & $19.36 \pm 0.04$ & \\
\hline 9 & & & $18.06 \pm 0.04$ & & $18.00 \pm 0.06$ & & & $18.01 \pm 0.04$ & \\
\hline 10 & & & - & & $15.54 \pm 0.06$ & & & - & \\
\hline 11 & & & $15.98 \pm 0.04$ & & $16.01 \pm 0.06$ & & & $15.97 \pm 0.04$ & \\
\hline 12 & & & $19.04 \pm 0.05$ & & $18.94 \pm 0.06$ & & & $18.99 \pm 0.04$ & \\
\hline
\end{tabular}

${ }^{a}$ Numbers atop the magnitude columns give the observations epochs in Modified Julian Days (MJD). Magnitude errors are given at $1 \sigma$ and include zero point errors, either absolute or relative, which are the dominant error source for the brightest objects.

Chandra positional uncertainty of 0.7 at $90 \%$ confidence level. Figure 5 shows a revised finding chart of the field around the updated X-ray position. The size of the X-ray error circle, 1'.2 $(99 \% \mathrm{cl})$, accounts for the overall accuracy of our astrometric calibration (see Sect. 2.2). The magnitudes of all objects detected within or close to the X-ray error circle are reported in Table 4. No other object has been detected down to the $3 \sigma$ limiting magnitudes of 21.54 and 21.00 for $H$ and $K^{\prime}$, respectively. All objects have been detected in both the $H$ and $K^{\prime}$ bands, except for \#9 and \#10. Figure 5 shows the position of these objects in the $\left(K^{\prime}, H-K^{\prime}\right)$ colour-magnitude diagram compared to other objects detected in the field. We note that object \#19 of Mereghetti et al. (2001), originally considered a potential candidate on the basis of the ROSAT X-ray position, lies more than two error radii away from the updated Chandra position. The CMD shows a well-defined main sequence extending up to $K^{\prime} \sim 12$, with the candidates occupying the fainter region and stretching over a broad range of colours.

By comparing our photometric results with those reported in literature for 1E 1841-045 (Durant 2005), it is evident that our source \#9 (source B in Durant 2005), shows a $\sim 3 \sigma$ variability
$\left(\Delta K_{\mathrm{s}}=0.9 \pm 0.3\right)$. As a comparison, the average $\Delta K_{\mathrm{s}}$ and $\Delta H$ between our photometry and that of Durant (2005) for 9 objects in the field of view is $\sim 0.2 \mathrm{mag}$. We then tentatively propose this object as the IR counterpart to this AXP.

\section{4. $S G R 1900+14$}

Figure 6 shows the NACO $K_{\mathrm{s}}$ band image around the SGR $1900+14$ radio position (RA $19^{\mathrm{h}} 07^{\mathrm{m}} 14^{\mathrm{s}} .33$, Dec $+09^{\circ} 19^{\prime} 20^{\prime} \cdot 1$, with a $1 \sigma$ uncertainty of $0 .^{\prime} 15$ in each coordinate; Frail et al. 1999). Our astrometry yields an overall $3 \sigma$ position uncertainty of 0.81 , which compares well with the one of 0.79 (99\% confidence level) quoted by Kaplan et al. (2002). We note that the PSFs of field objects are slightly elongated in the NACO images, and one of the objects detected within the radio error circle (our \#3 and object $\mathrm{C}$ of Kaplan et al. 2002) appears extended. However, its PSF is consistent with what is measured for most field objects. In this case, photometric calibration was performed using as reference the magnitudes of a few objects detected around the target position (Kaplan et al. 2002; Frail et al. 1999) (see Fig.6). The three 

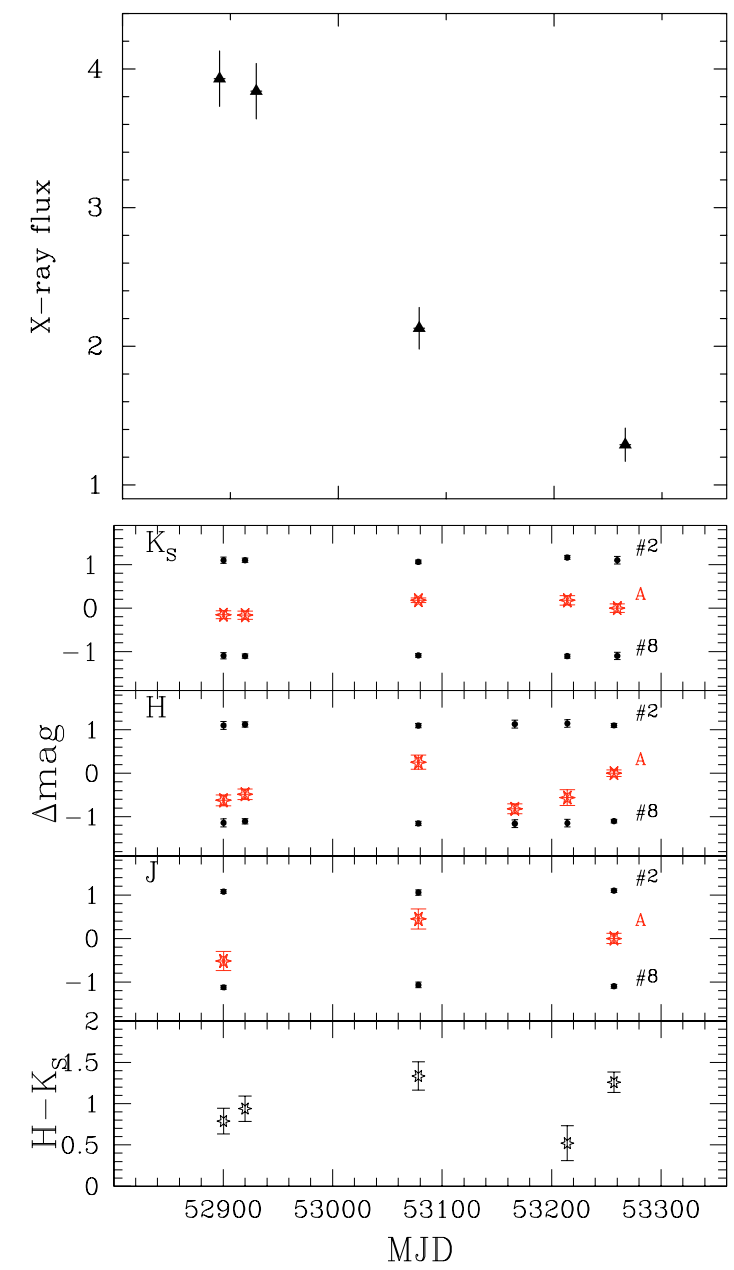

Fig. 2. XTEJ1810-197: Top panel: X-ray flux (shown in units of $10^{-11} \mathrm{erg} \mathrm{cm}^{-2} \mathrm{~s}^{-1}$ ) history in the $0.5-10 \mathrm{keV}$ energy range (from Gotthelf \& Halpern 2007). Lower panels: from top to bottom: $K_{\mathrm{s}}, H$, and $J$ band relative magnitude variation for XTEJ1810-197 counterpart (red star) and for two nearby, bright comparison objects. Magnitude variations are computed relative to the 2004 September observation. To fit in the plot better, magnitude differences for objects \#2 and \#8 have been shifted by +1.1 and -1.1 mag, respectively. Bottom panel shows the $H-K_{\mathrm{s}}$ colour variation. Magnitude errors at $(1 \sigma)$ are shown. These include internal errors, aperture correction errors, and zero point errors.

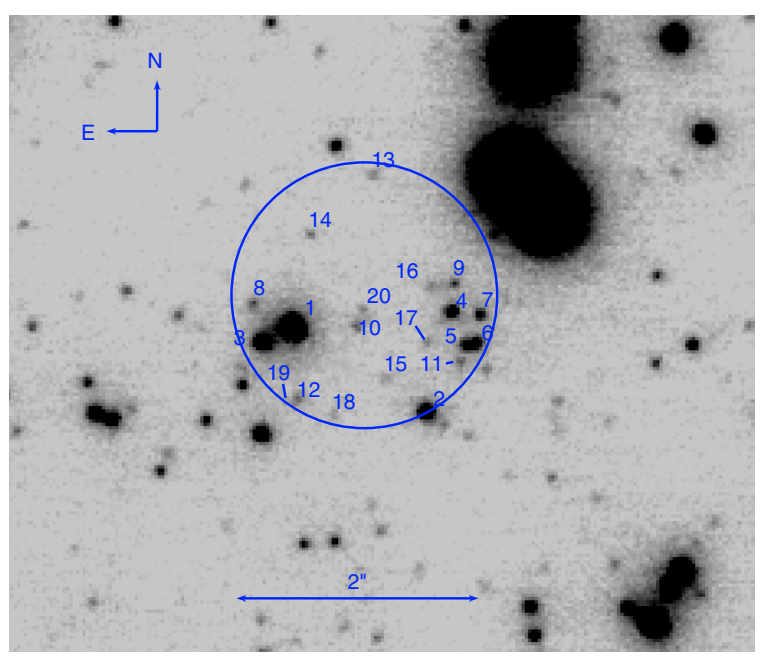

Fig. 3. 1RXS J1708-4009: NACO $K_{\mathrm{s}}$-band image of the source field with the 1". 1 radius (99\% confidence level) X-ray error circle overlaied. Objects detected at $\geq 3 \sigma$ are labeled.
Table 3. 1RXS J1708-4009: $J, H$, and $K_{\mathrm{s}}$ magnitudes for all the objects detected within $11^{\prime \prime} 1$ of the source X-ray position, ordered by decreasing $K_{\mathrm{s}}$ magnitude.

\begin{tabular}{cccccc}
\hline \hline Id & RA $\left(^{\circ}\right)$ & Dec $\left(^{\circ}\right)$ & $J$ & $H$ & $K_{\mathrm{s}}{ }^{a}$ \\
\hline 1 & 257.19551 & -40.14796 & $20.93 \pm 0.09$ & $18.60 \pm 0.06$ & $17.53 \pm 0.04$ \\
2 & 257.19510 & -40.14815 & $22.23 \pm 0.17$ & $19.84 \pm 0.09$ & $18.81 \pm 0.05$ \\
3 & 257.19560 & -40.14799 & $22.11 \pm 0.25$ & $20.01 \pm 0.12$ & $18.85 \pm 0.05$ \\
4 & 257.19503 & -40.14792 & $22.03 \pm 0.17$ & $20.19 \pm 0.09$ & $19.63 \pm 0.07$ \\
5 & 257.19498 & -40.14800 & - & $20.50 \pm 0.10$ & $19.95 \pm 0.08$ \\
6 & 257.19495 & -40.14800 & - & - & $20.03 \pm 0.09$ \\
7 & 257.19494 & -40.14793 & - & $20.90 \pm 0.11$ & $20.25 \pm 0.08$ \\
8 & 257.19563 & -40.14790 & - & - & $20.83 \pm 0.10$ \\
9 & 257.19502 & -40.14786 & - & $21.55 \pm 0.18$ & $20.92 \pm 0.11$ \\
10 & 257.19531 & -40.14795 & - & - & $20.94 \pm 0.11$ \\
11 & 257.19500 & -40.14804 & - & - & $20.69 \pm 0.10$ \\
12 & 257.19548 & -40.14812 & - & - & $21.12 \pm 0.19$ \\
13 & 257.19526 & -40.14761 & - & - & $21.23 \pm 0.14$ \\
14 & 257.19545 & -40.14774 & - & - & $21.34 \pm 0.21$ \\
15 & 257.19523 & -40.14807 & - & - & $21.52 \pm 0.17$ \\
16 & 257.19509 & -40.14787 & - & - & $21.58 \pm 0.12$ \\
17 & 257.19510 & -40.14799 & - & - & $21.59 \pm 0.16$ \\
18 & 257.19538 & -40.14816 & - & $21.34 \pm 0.15$ & $21.66 \pm 0.14$ \\
19 & 257.19550 & -40.14813 & - & - & $21.74 \pm 0.16$ \\
20 & 257.19530 & -40.14792 & - & - & $21.88 \pm 0.19$ \\
\hline
\end{tabular}

${ }^{a} \overline{\text { No object has been detected in the } L^{\prime} \text { band. Magnitude errors are given }}$ at $1 \sigma$, and include zero point uncertainties.

new objects detected in our NACO images are also marked (\#7, 8, and 9). In particular, object \#7 falls very close to the position of \#3, which prompted us to use PSF photometry rather than aperture photometry as done, instead, for the other NACO observations. The magnitudes of all the detected objects are listed in Table 5. No other object is detected close to or within the radio position error circle down to a $3 \sigma$ limiting magnitude of $K_{\mathrm{s}}=22.60$ and 22.25 for the March 31 and for the July 20 observations, respectively. We note that among our newly detected objects, \#7 $\left(K_{\mathrm{s}} \sim 19.7\right)$ is the only new source within the $99 \%$ error circle, and it displays some evidence of variability $\left(\Delta K_{\mathrm{s}}=0.47 \pm 0.11\right)$ between the two epochs. Unfortunately, its proximity to object \#3 prevents a better determination of the actual magnitude variation, even through PSF photometry. As we did in Sect. 3.1, we evaluated the effects of random errors on our relative photometry by measuring the magnitude scatter of a number of test stars of brightness comparable to object \#7 and selected it close to the field centre in order not to introduce biases into our PSF photometry due to the position-dependent NACO PSF. We found that, for the test stars, the scatter is $\approx 0.1 \mathrm{mag}$. Even adding the random error we found that the measured variation is still significant, although only at the $\sim 3 \sigma$ level. Based on variability, we then propose object \#7 as a tentative IR candidate counterpart to SGR $1900+14$.

\section{Discussion}

Before discussing our results, we would like to clarify here how we define a reliable identification of an IR counterpart to an AXP or SGR. All of the current well-defined counterparts have been established either through a very accurate positional coincidence (no other candidates in the positional error circle) or by the detection of significant IR variability. In some cases, what are usually defined (not very clearly) as "strange" colours with respect to the other stars in the field of view has been used as a method to claim the detection of a counterpart. However, we prefer not 

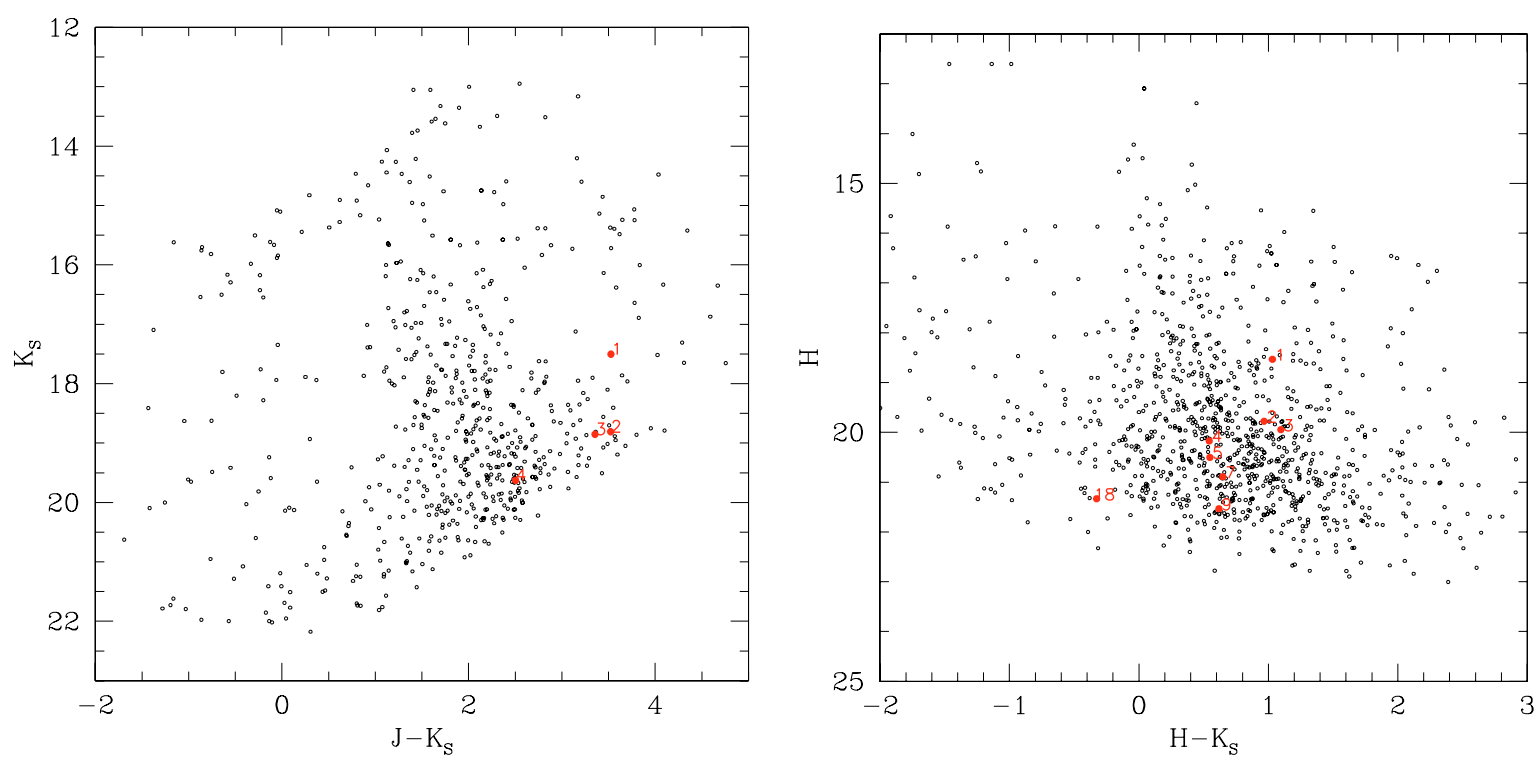

Fig. 4. 1RXS J1708-4009: colour-magnitude diagrams for the objects in the field of view. Sources detected within or close to the X-ray error circle (see Fig. 3) are shown in red and labelled.
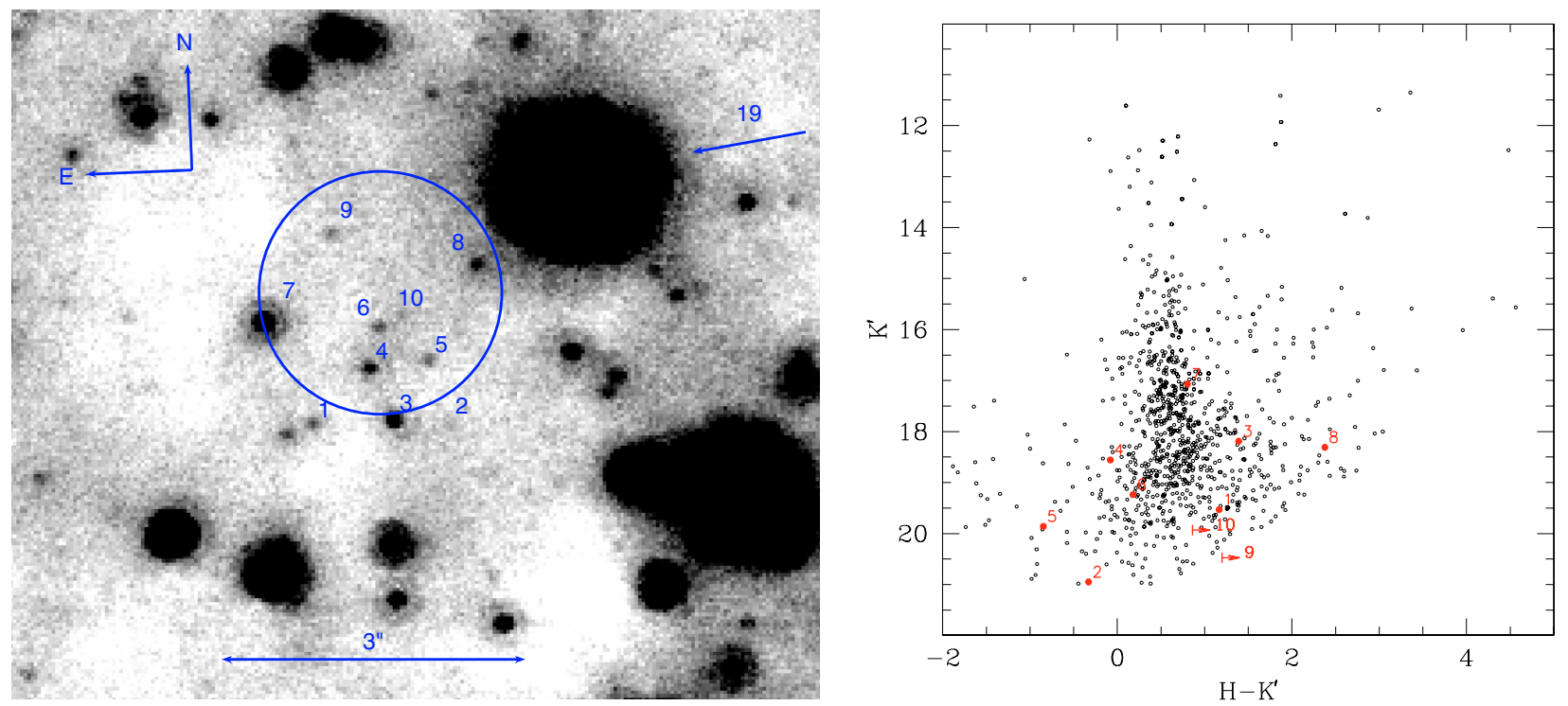

Fig. 5. Left panel: 1E 1841-045: CFHT $K^{\prime}$-band image of field, with the 1'.2 radius (99\% confidence level) X-ray error circle overlaied. Objects detected at $\geq 3 \sigma$ are labeled. Object \#19 of Mereghetti et al. (2001) is labeled as a reference. Right panel: $K^{\prime}, H-K^{\prime}$ CMD for 1E $1841-045$ field. Objects detected within or close to the X-ray error circle (left) are shown in red and labelled.

to adopt this "colour" criterion because we find it rather misleading. In fact, i) almost all counterparts reliably identified through IR variability detection or positional coincidence have colours that are not much different from some of the their field stars; and ii) our current lack of knowledge of the exact physics behind the IR emission of AXPs and SGRs does not allow any prediction of their IR spectra. Another criterion often used to propose or strengthen an identification is the X/IR flux ratio. We find this method rather rough and not constraining at all for the following reasons: i) AXPs and SGRs are variable both in the X-ray and IR bands, and we do not have a clear idea yet of the connection between these two bands; ii) there are no theoretical predictions with respect to the X/IR ratio that an AXP or SGR is expected to have; and iii) with the exception of their IR variability, the IR counterpart to these neutron stars are, at our current knowledge, consistent with the IR emission of very low-mass stars, which if found in the source error circle will then result in the same X/IR ratio. This is why, as long as significant variability is not detected and if more than one faint IR object is present in the positional circle, any proposed IR conterpart needs a further confirmation.

\subsection{XTEJ1810-197 IR variability}

The IR observations with the VLT-NACO camera of XTEJ1810-197 performed after the outburst, unveiled its IR counterpart (Israel et al. 2004b). Follow-up observations performed six months later detected IR variability from the candidate counterpart (Rea et al. 2004), with a flux decrease by about a factor of 2. Simultaneous X-ray observations over the same period revealed a similar X-ray flux decrease between the two epochs (Rea et al. 2004; Gotthelf et al. 2004). This 
Table 4. 1E 1841-045: $H$ and $K^{\prime}$ magnitudes for all the objects detected within 1 .' 1 of the source X-ray position.

\begin{tabular}{lcccc}
\hline \hline Id. & ${\text { RA }\left({ }^{\circ}\right)}$ & Dec $\left(^{\circ}\right)$ & $H$ & $K^{\prime a}$ \\
\hline 1 & 280.330811 & -4.936699 & $20.70 \pm 0.31$ & $19.53 \pm 0.15$ \\
2 & 280.330444 & -4.936720 & $20.62 \pm 0.29$ & $20.95 \pm 0.49$ \\
3 & 280.330597 & -4.936695 & $19.58 \pm 0.10$ & $18.19 \pm 0.03$ \\
4 & 280.330658 & -4.936555 & $18.48 \pm 0.03$ & $18.56 \pm 0.05$ \\
5 & 280.330475 & -4.936538 & $19.01 \pm 0.06$ & $19.86 \pm 0.18$ \\
6 & 280.330627 & -4.936442 & $19.42 \pm 0.08$ & $19.24 \pm 0.11$ \\
7 & 280.330933 & -4.936414 & $17.88 \pm 0.02$ & $17.07 \pm 0.02$ \\
8 & 280.330353 & -4.936280 & $20.69 \pm 0.29$ & $18.31 \pm 0.04$ \\
9 & 280.330760 & -4.936186 & $>21.54$ & $20.47 \pm 0.28$ \\
10 & 280.330670 & -4.936334 & $>21.54$ & $19.93 \pm 0.16$ \\
\hline
\end{tabular}

${ }^{a}$ Magnitude errors are given at $1 \sigma$ confidence level.

suggested that the correlated IR/X-ray variability is a characteristic of this source, as expected if the IR emission were dominated by the reprocessing of the X-ray emission from a fossil disk around the magnetar (Perna et al. 2000a; Perna \& Hernquist 2000b). However, our recent IR observations show that such a correlation is not as obvious as thought before (see also Camilo et al. 2007b). While the IR flux is indeed variable, it does not follow the same monotonic decrease in the X-ray flux observed in the post-outburst phase (see Fig. 2; and Gotthelf \& Halpern 2007). Instead, the IR variability appears more like the one observed in radio band (Camilo et al. 2006, 2007c). Unfortunately, the IR observations are too sparse for any firm conclusion to be drawn regarding a possible connection between the radio and the IR variability. Furthermore, we note that the onset of the radio emission (January 2005; Camilo et al. 2006) occurred after our IR observations.

In the fossil-disk scenario, the XTE J1810-197 IR variability can hardly be explained if the IR emission were dominated by reprocessing of the X-ray radiation from the pulsar. In this case in fact, the IR flux should decrease as the X-ray flux drops (see Rea et al. 2004, for details), and no increase in any of the IR bands would be expected. If, on the other hand, the IR flux were dominated by the disk emissivity resulting from viscous dissipation (which could be the case if the disk inner radius is farther out), then flux variability would be the result of a variation in the disk mass-inflow rate. Given the non-correlated IR and X-ray variability we observe, this scenario could be more viable. However, while a variability in the IR emission is easily predictable as resulting from a mass-inflow rate variation, this should take place in the same way in all the IR bands. As noted in Sect. 3.1, we see a hint of an IR-band dependent variability, although no significant conclusion can be drawn on this aspect yet.

Within the magnetars scenario, a few different models have been proposed for the production of the IR radiation (e.g. Heyl \& Hernquist 2005; Beloborodov \& Thompson 2007), but none of them makes specific predictions (yet) for the IR variability that would permit a direct comparison with our data.

IR variability has been observed for other magnetars, i.e. 1E 1048.1-5937 (Israel et al. 2002; Tam et al. 2007), 4U 0142+614 (Hulleman et al. 2004), SGR 1806-20 (Israel et al. 2005), and 1E2259+586 (Tam et al. 2004). Unfortunately, with the current data no firm conclusion can be made yet about the IR variability in connection with variabilities in other observing bands, except for 1E 2259+586 and SGR 1806-20 (Tam et al. 2004; Israel et al. 2005), where a correlation with the source bursting activity and X-ray flux enhancement has been detected.

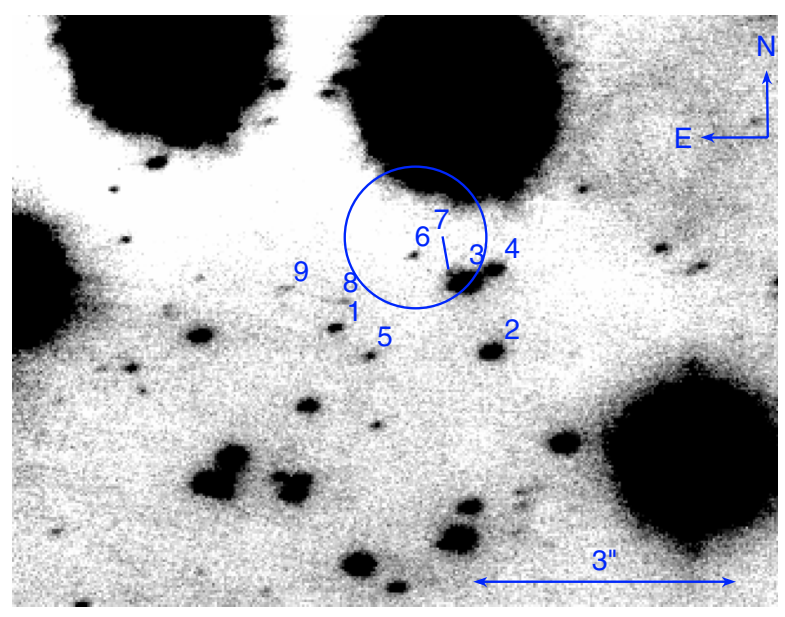

Fig. 6. SGR $1900+14$. Zoom-in of the area of the $K_{\mathrm{s}}$ band image of 2006 March 31 around the radio position of Frail et al. (1999). The error circle (radius 0.81 at 99\% confidence level) is shown. Two large ghosts are visible atop the position of SGR $1900+14$, hiding star E of Kaplan et al. (2002).

Table 5. SGR 1900+14: $K$ magnitudes of the March and July 2006 observations.

\begin{tabular}{lcccc}
\hline \hline Id. & ${\text { RA }\left({ }^{\circ}\right)}$ & $\operatorname{Dec}\left(^{\circ}\right)$ & $K(2006.03 .31)$ & $K(2006.07 .20)^{a}$ \\
\hline 1 & 286.81000 & 9.32198 & $19.36 \pm 0.09$ & $19.39 \pm 0.06$ \\
2 & 286.80949 & 9.32190 & $18.14 \pm 0.07$ & $18.05 \pm 0.05$ \\
3 & 286.80957 & 9.32212 & $17.31 \pm 0.07$ & $17.25 \pm 0.05$ \\
4 & 286.80948 & 9.32216 & $18.42 \pm 0.07$ & $18.48 \pm 0.05$ \\
5 & 286.80989 & 9.32189 & $20.10 \pm 0.11$ & $19.96 \pm 0.08$ \\
6 & 286.80974 & 9.32220 & $20.73 \pm 0.26$ & $20.85 \pm 0.22$ \\
7 & 286.80963 & 9.32215 & $19.21 \pm 0.08$ & $19.68 \pm 0.08$ \\
8 & 286.80997 & 9.32206 & $20.86 \pm 0.19$ & $20.55 \pm 0.13$ \\
9 & 286.81016 & 9.32210 & $20.59 \pm 0.20$ & $20.46 \pm 0.15$ \\
\hline
\end{tabular}

${ }^{a}$ Errors on the magnitudes are given at $1 \sigma$ confidence level.

\subsection{Other sources: 1E 1841-045, SGR 1900+14, and 1RXS J1708-4009}

No IR counterpart had been claimed so far for 1E 1841-045 and SGR $1900+14$, probably because of the crowding of the field in which these magnetars happen to lie. By comparing our photometric results with those reported in the literature for 1E 1841045 , it is evident that there is one object, our source \#9 (source B in Durant 2005), with magnitudes similar to the other AXP counterparts and showing a $\sim 3 \sigma$ variability. Furthermore, comparing our two observations of SGR $1900+14$ we find a $\sim 3 \sigma$ variability in source \#7 (not detected by Kaplan et al. 2002) because it was too faint for their limiting magnitudes). We therefore consider these objects as possible IR counterparts to these two neutron stars.

The new deep observation of 1RXS J1708-4009 show 20 sources within the $99 \%$ X-ray error circle and in its vicinity (see Fig. 3). None of these objects showed significant variability compared to other observations in the literature, which could help identifying them as reliable magnetar counterparts.

Note that the magnitudes of both the previous candidates (our \#1 and \#3; Israel et al. 2003; Safi-Harb \& West 2005; Durant \& van Kerkwijk 2006) would make 1RXS J1708-4009 much brighter in the IR band than any other AXP (see e.g. Israel et al. 2004a). Furthermore, all the other fainter candidates we report here, all within the source positional errors (see Fig. 3), have brightness that agree better with the IR emission properties of 
AXPs than do both objects \#1 and \#3. Durant \& van Kerkwijk (2006) proposed source \#3 as a possible candidate on the basis of IR variability, the strange colours, and the resulting $\mathrm{X} / \mathrm{IR}$ ratio of this object. We believe that given the large number of faint IR sources lieing within the 1RXS J1708-4009 positional error circle, and the rather bright magnitudes of source \#3, neither the $\sim 2.5 \sigma$ IR variability, its colours (see Fig. 4), nor its $\mathrm{X} / \mathrm{IR}$ ratio can reliably identify this object as the AXP (see also above). We would like to stress that we cannot exclude any of these candidates, but a firm identification of an IR counterpart to 1RXS J1708-4009 is far from being a settled issue.

This AXP has recently been observed to have a variable X-ray emission (Rea et al. 2005, 2007b; Campana et al. 2007), which if correlated somehow with the IR as for 1E 2259+586 (Tam et al. 2004), would imply a variable IR counterpart. In particular, the X-ray variability observed for 1RXS J1708-4009 in sparse X-ray observations over several years was of the order of $50 \%$, which would roughly imply (if X-ray and IR are directly correlated) an IR variability of $\sim 0.5 \mathrm{mag}$, not detected from any of the objects near the position of 1RXS J1708-4009. However, the lack of simultaneous IR and X-ray observations of this source prevent us from drawing any firm conclusions about possible correlated variability.

\section{Conclusions}

The IR counterpart to XTE J1810-197 is confirmed to be variable in time (as previously proposed by Rea et al. 2004). However, despite previous claims, its variability might be more like its radio behaviour than to its X-ray variability, which appears to decay smoothly after the outburst. Simultaneous IR and radio observations of this AXP are needed to reliably assess this possibility.

To date, IR counterparts to AXPs and SGRs have only been confirmed for XTE J1810-197 (Israel et al. 2004b), 1E 1048.15937 (Wang et al. 2002; Israel et al. 2002), 1E 2259+586 (Hulleman et al. 2001), 4U0142+614 (Hulleman et al. 2000), and SGR 1806-20 (Israel et al. 2005; Kosugi et al. 2005). For the AXP 1E 1841-045 and SGR 1900+14, we propose here two possible candidates based on the detection of IR variability. For all the remaining AXPs and SGRs, we still miss a candidate or confirmed IR counterpart (see, e.g., Wachter et al. 2004; Durant 2005; Durant \& van Kerkwijk 2007; Muno et al. 2006; Gelfand \& Gaensler 2007).

Both our results and others for optical and IR observations of AXPs and SGRs show that we are still far from having an overall picture of the optical and IR behaviour of these neutron stars. Further observations, possibly simultaneously in a wide energy range, are needed to refine current theoretical models and shed light on the optical and IR emission mechanisms of AXPs and SGRs.

Acknowledgements. Based on observations collected at the European Southern Observatory, Paranal, Chile, under programme ID 071.D-0503, 072.D-0297, 073.D-0381, 076.D-0383, 277.D-5005, at the Canada-France-Hawaii Telescope (CFHT), which is operated by the National Research Council of Canada, the Institut National des Sciences de l'Univers of the Centre National de la Recherche Scientifique of France, and the University of Hawaii, and at the Gemini Observatory, which is operated by the Association of Universities for Research in Astronomy Inc., under a cooperative agreement with the NSF on behalf of the Gemini partnership: the National Science Foundation (United States), the Science and Technology Facilities Council (United Kingdom), the National Research Council (Canada), CONICYT (Chile), the Australian Research Council (Australia), CNPq (Brazil) and CONICET (Argentina). This publication makes use of data products from the Two Micron All Sky Survey, which is a joint project of the University of Massachusetts and the Infrared Processing and Analysis Center/California Institute of Technology, funded by the National Aeronautics and Space Administration and the National Science Foundation. We thank the Yepun (UT4) and NACO team for their constant help on the observation optimization during the 3 years covered by this programme, and the referee for his/her very careful reading of the paper and constructive report. V.T. and G.L.I. acknowledge support from MIUR funds. N.R. is supported by NWO Veni Fellowship. S.Z. acknowledges STFC (ex-PPARC) for support through an Advanced Fellowship.

\section{References}

Baring, M. G., \& Harding, A. K. 1998, ApJ, 507, L55

Beloborodov, A., \& Thompson, C. 2007, ApJ, 657, 967

Bertin, E., \& Arnouts, S. 1996, A\&AS, 117, 393

Burgay, M., Rea, N., Israel, G. L., et al. 2006, MNRAS, 372, 410

Burgay, M., Rea, N., Israel, G. L., et al. 2007, Astronomers' Telegram, \# 903

Camilo, F., Ransom, S., Halpern, J., et al. 2006, Nature, 442, 892

Camilo, F., Ransom, S. M., Halpern, J. P., Reynolds, J. 2007a, ApJ, 666, L93

Camilo, F., Ransom, S. M., Penalver, J., et al. 2007b, ApJ, 663, 497

Camilo, F., Cognard, I., Ransom, S., et al. 2007c, ApJ, 663, 497

Campana, S., Rea, N., Israel, G. L., et al. 2007, A\&A, 463, 1047

Chatterjee, P., Hernquist, L., \& Narayan, R. 2000, ApJ, 534,

Duncan, R. C., \& Thompson, C. 1992, ApJ, 392, L9

Durant, M. 2005, ApJ, 632, 563

Durant, M., \& van Kerkwijk, 2005, ApJ, 628, L135

Durant, M., \& van Kerkwijk 2006, ApJ, 648, 534

Durant, M., \& van Kerkwijk 2007, ApJ, in press [arXiv: 0711.3985]

Frail, D. A., Kulkarni, S. R., Bloom, J. S. 1999, Nature, 398, 127

Gelfand, J. D., \& Gaensler, B. M. 2007, ApJ, 667, 1111

Götz, D., Mereghetti, S., Tiengo, A., Esposito, P. 2006, A\&A, 449, L31

Gotthelf, E. V., \& Halpern, J. P. 2007, Ap\&SS, 308, 79

Gotthelf, E. V., Halpern, J. P., Buxton, M., \& Bailyn, C. 2004, ApJ, 605, 368

Halpern, J., Gotthelf, E. V., Becker, R. H, et al. 2005, ApJ, 632, L29

Heyl, J. S., \& Hernquist, L. 2005, MNRAS, 362, 777

Hulleman, F., van Kerkwijk, M. H., \& Kulkarni, S. R. 2000, Nature, 408, 68

Hulleman, F., Tennant, A. F., van Kerkwijk, M. H., et al. 2001, ApJ, 563, L49

Hulleman, F., van Kerkwijk, M. H., \& Kulkarni, S. R. 2004, A\&A, 416, 1037

Israel, G. L., Covino, S., Stella, L., et al. 2002, ApJ, 580, L143

Israel, G. L., Covino, S., Perna, R., et al. 2003, ApJ, 589, L93

Israel, G. L., Stella, L., Covino, S., et al. 2004a, IAU Symp. 218, ed. F. Camilo, \& B. M. Gaensler, 247

Israel, G. L., Rea, N., Mangano, V., et al. 2004b, ApJ, 603, L97

Israel, G. L., Covino, S., Mignani, R., et al. 2005, ApJ, 438, L1

Kaplan, D. L., Kulkarni, S. R., Frail, D. A., \& van Kerkwijk, M. H. 2002, ApJ,

566,378

Kosugi, G., Ogasawara, R., \& Terada, H. 2005, ApJ, 623, L125

Kuiper, L., Hermsen, W., \& Méndez, M. 2004, ApJ, 613, 1173

Kuiper, L., Hermsen, W., den Hartog, P., \& Collmar, W. 2006, ApJ, 645, 556

Leggett, S. K., Hawarden, T. G., Currie, J. M., et al. 2003, MNRAS, 345, 144

Mereghetti, S., Mignani, R., Covino, S., et al. 2001, MNRAS, 321, 143

Mereghetti, S., Götz, D., Mirabel, I. F., Hurley, K. 2005, A\&A, 433, L9

Muno, M. P., Clark, J. S., Crowther, P. A., et al. 2006, ApJ, 636, L41

Perna, R., \& Hernquist, L. 2000, ApJ, 544, L57

Perna, R., Hernquist, L., \& Narayan, R. 2000, ApJ, 541, 344

Persson, S. E., Murphy, D. C., Krzeminski, W., et al. 1998, AJ, 116, 2475

Rea, N., Testa, V., Israel, G. L., et al. 2004, A\&A, 425, L5

Rea, N., Oosterbroek, T., Zane, S., et al. 2005, MNRAS, 361, 710

Rea, N., Turolla, R., Zane, S., et al. 2007a, ApJ, 661, L65

Rea, N., Israel, G. L., Oosterbroek, T., et al. 2007b, Ap\&SS, 308, 505

Safi-Harb, S., \& West, J. 2005, AdSpR, 35, 1172

Skrutskie, M., Cutri, R. M., Stiening, R., et al. 2006, AJ, 131, 1163

Stetson, P. B. 1992, Astronomical Data Analysis Software and Systems I, ASP Conf. Ser., 25, 297

Tam, C. R., Kaspi, V. M., van Kerkwijk, M. H., Durant, M. 2004, ApJ, 617, L53

Tam, C. R., Gavriil, F. P., Dib, R., et al. 2007, ApJ, in press [arXiv: 0707.2093]

Thompson, C., \& Duncan, R. C. 1995, MNRAS, 275, 255

Wachter, S., Patel, S., Kouveliotou, C., et al. 2004, ApJ, 615, 887

Wang, Z., \& Chakrabarty, D. 2002, ApJ, 579, L33

Wang, Z., Chakrabarty, D., \& Kaplan, D. L. 2006, Nature, 440, 772

Woods, P., \& Thompson, C. 2006, Compact stellar X-ray sources, ed. W. Lewin, \& M. van der Klis (Cambridge University Press)

[arXiv: astro-ph/0406133] 